S5 Appendix. Full search strategy used for the 'Global Health' electronic database.

\title{
1) Sexual and Reproductive Health and Rights
}

\begin{tabular}{|l|l|}
\hline Keywords: & $\begin{array}{l}\text { "sexual health" OR "sexual rights" OR "reproductive health" OR } \\
\text { "reproductive rights" OR SRHR OR SRH OR contracepti* OR } \\
\text { pregnan* OR "maternal health" OR maternity OR antenatal OR } \\
\text { postnatal OR obstetric OR delivery OR aborti* OR post-aborti* } \\
\text { OR "family planning" OR "sexually transmitted" OR STI OR } \\
\text { STD OR HIV OR "gender-based violence" OR "intimate partner } \\
\text { violence" OR "sexual abuse" OR "female genital" OR FGM OR } \\
\text { "female circumcision" }\end{array}$ \\
\hline $\begin{array}{l}\text { Controlled } \\
\text { vocabulary: }\end{array}$ & $\begin{array}{l}\text { sexual health/ OR reproductive health/ OR contraceptives/ OR } \\
\text { contraception/ OR pregnancy/ OR pregnant adolescents/ OR } \\
\text { maternity/ OR prenatal care/ obstetrics/ OR abortion/ OR induced } \\
\text { abortion/ OR family planning/ OR sexually transmitted diseases/ } \\
\text { OR human immunodeficiency viruses/ OR HIV infections/ OR } \\
\text { domestic violence/ OR spouse abuse/ OR sexual abuse/ }\end{array}$ \\
\hline \multicolumn{1}{|c|}{ AND } \\
\hline
\end{tabular}

\section{2) Barriers/Facilitators/Interventions}

\begin{tabular}{|c|c|}
\hline Keywords: & $\begin{array}{l}\text { barrier* OR facilitator* OR intervention* OR service* OR } \\
\text { program* OR information OR education* }\end{array}$ \\
\hline $\begin{array}{l}\text { Controlled } \\
\text { vocabulary: }\end{array}$ & $\begin{array}{l}\text { social barriers/ OR intervention/ OR health services/ OR } \\
\text { maternity services/ OR medical services/ OR public health } \\
\text { services/ OR services/ OR social services/ OR programs/ OR } \\
\text { program evaluation/ OR information/ OR education/ OR } \\
\text { education programmes/ OR health education/ OR medical } \\
\text { education/ OR sex education/ OR prenatal education/ }\end{array}$ \\
\hline \multicolumn{2}{|r|}{ AND } \\
\hline \multicolumn{2}{|r|}{ 3) Population: Young People (10-24 years) } \\
\hline Keywords: & adolescen* OR young OR youth \\
\hline $\begin{array}{l}\text { Controlled } \\
\text { vocabulary: }\end{array}$ & adolescents/ OR youth/ \\
\hline \multicolumn{2}{|r|}{ AND } \\
\hline \multicolumn{2}{|r|}{ 4) Population: Refugees, Asylum Seekers } \\
\hline Keywords: & $\begin{array}{l}\text { migrant OR migrants OR migration OR refugee* OR "asylum } \\
\text { seekers" }\end{array}$ \\
\hline $\begin{array}{l}\text { Controlled } \\
\text { vocabulary: }\end{array}$ & migrants/ OR migration/ OR refugees/ \\
\hline
\end{tabular}

Filters: English Language, Publication Year (2008-current) 